'Instituto de Sociología. Facultad de Ciencias Sociales. Pontificia Universidad Católica de Chile. Santiago, Chile. aSocióloga bPhD.

Trabajo no recibió financiamiento. Los autores declaran no tener conflictos de interés.

Recibido el 9 de julio de 2019, aceptado el 19 de diciembre de 2019.

Correspondencia a: María Beatriz Fernández Lorca Pontificia Universidad Católica de Chile, Instituto de Sociología, Vicuña Mackenna 4680, Macul, Santiago de Chile. mrfernan@uc.cl

\section{El efecto del cuidado informal en la salud de los cuidadores familiares de personas mayores dependientes en Chile}

\author{
M. BEATRIZ FERNÁNDEZ ${ }^{1, \mathrm{a}, \mathrm{b}}, \mathrm{M}$. SOLEDAD HERRERA ${ }^{1, \mathrm{a}, \mathrm{b}}$
}

\section{Health effects of dependent older people caregiving by family members}

Background: Care required by dependent older people is mostly provided by close family members. Caregiving is a demanding task, negatively affecting the physical and mental health of the caregiver. Aim: To describe the sociodemographic characteristics of informal caregivers of dependent elderly people in Chile and to evaluate the effect of providing care on the health of caregivers. Material and Methods: Data from the Economic Characterization Survey (CASEN) done in 2015 was used. Using as index cases older people who were receiving care from a family member, 4,313 informal caregivers were identified, whose characteristics were analyzed. Descriptive statistics and matching through propensity score were estimated, based on a logistic model, which included covariates such as sex, age, marital status, education, occupation, and income. Finally, the effect of being an informal caregiver versus not being a caregiver on the perception of health was calculated. Results. Forty four percent of the caregivers were offspring and $33.6 \%$ couples. Sixty seven percent were women, with a mean age of 56.5 years. Half of them had a partner, $43 \%$ had basic education or less, and $61 \%$ were inactive. Being a caregiver had a significant effect of health perception. Conclusions: People belonging to certain more vulnerable groups are more likely to assume caregiving. Providing care to a dependent older person has a negative effect on health perception.

(Rev Med Chile 2020; 148: 30-36)

Key words: Aged; Caregivers; Patient Care. hile posee actualmente $17,5 \%$ de personas de 60 años y más ${ }^{1}$, quienes, en promedio, pueden alcanzar una expectativa de vida cercana a los 81 años $^{2}$. Este escenario demográfico conlleva diversos desafíos, uno de los cuales se asocia al fenómeno de la dependencia funcional, especialmente entre los más envejecidos. Según datos nacionales, el 14,4\% de la población mayor es dependiente, cifra que se incrementa a casi $40 \%$ en la población de 80 y más años ${ }^{1}$.

La mayoría de los cuidados requeridos por este grupo son provistos por los familiares más cercanos al interior del hogar-pareja e hijos-, lo que se explica por la preferencia de los dependientes a permanecer en sus propias residencias, al alto costo de los cuidados formales y la relevancia de la familia en la sociedad latinoamericana, observándose, además, una carencia de estructuras alternativas provistas por el Estado o el mercado ${ }^{3}$.

Por lo general, los cuidadores informales no reciben remuneración económica por esta tarea ${ }^{4}$, ni tampoco cuentan con la formación para los diferentes cuidados que proveen ${ }^{5}$. Así, el cuida- 
do puede llegar a convertirse en una tarea muy exigente, provocando tensión física, fatiga, desgaste emocional o estrés ${ }^{6,7}$, lo que en situaciones extremas puede dar paso al llamado "síndrome del cuidador", donde el individuo se siente incapaz de afrontar todas las responsabilidades que le exige el cuidado, llegando a una situación de sobrecarga que compromete su bienestar, y que puede desencadenar alteraciones en su salud física y emocional ${ }^{8}$.

En esta línea, varios son los estudios que se han realizado para determinar si proveer cuidado informal se asocia negativamente con la salud tanto física como mental del cuidador, lo cual ha sido ampliamente confirmado por varios de estos estudios, destacando algunas revisiones sistemáticas y metaanálisis efectuados ${ }^{6,9,10}$. En el caso particular de América Latina, si bien la evidencia existente es aún escasa, esta también sugiere que el cuidado compromete la salud de quien cuida ${ }^{5,11-14}$. Ahora bien, uno de los problemas que se suscita al intentar estudiar la relación entre cuidado informal y salud, es que ser cuidador no es algo que se dé aleatoriamente en la población, existiendo un sesgo de selección ${ }^{6,15,16}$. Ser cuidador depende, entre otras cosas, de la habilidad del potencial cuidador para proveer cuidado, o del tiempo disponible para realizar esta tarea. Ello sugiere, por ejemplo, que las mujeres o personas con menor nivel socioeconómico-quienes a su vez están asociados con peores niveles de salud-tengan mayor probabilidad de desempeñar este rol $^{15}$.

Algunos estudios recientes han utilizado métodos cuasi experimentales- tales como análisis de propensity score, regresiones discontinuas, modelos de diferencia en diferencia, entre otros- para eliminar el sesgo de selección en las estimaciones del efecto del cuidado causado por factores no observados y así permitir la inferencia causal ${ }^{16-20}$. Es decir, estos métodos permiten asegurar que el efecto del cuidado en la salud es causado netamente por la entrega de este cuidado y no por otros factores ${ }^{21}$.

En este contexto, el objetivo de este estudio es doble. Por una parte, se busca describir las características sociodemográficas de los cuidadores informales de personas mayores en Chile, para luego, mediante el método de matching a través de propensity score, evaluar el efecto de proveer cuidado informal en la salud de los cuidadores.

\section{Material y Método}

\section{Fuente de datos}

El análisis estadístico se realizó en base a los datos de la Encuesta de Caracterización Socioeconómica del año 2015 (CASEN). Esta encuesta fue aplicada a un total de 83.887 hogares residentes en 324 comunas de las 15 regiones del país, recolectando información sobre 266.968 personas.

CASEN ha incorporado preguntas que permiten caracterizar la dependencia funcional, basada en las actividades básicas e instrumentales de la vida diaria. Asimismo, incluye la pregunta s35a. "Debido a su estado de salud, ¿alguna persona del hogar le presta ayuda para realizar esta(s) actividad(es)?". En base a ellas, se identificaron como cuidadores informales de personas mayores a todo individuo de 15 años o más que se identificaba como quien presta ayuda a una persona en el hogar de 60 años o más, calificado como dependiente funcional. En términos numéricos, de las 49.534 personas de 60 años o más incluidas en CASEN 2015, 7.098 fueron clasificadas con algún nivel de dependencia. De este total, solamente 5.099 mencionaron recibir ayuda por parte de algún miembro del hogar mayor de 15 años (cuidador informal). Posteriormente, se chequeó la información disponible de estos cuidadores informales, reduciéndose finalmente la muestra a 4.313 casos. Específicamente se eliminaron los casos en que la persona mayor dependiente se identificaba a sí misma como quien se presta ayuda. También se excluyeron los casos donde la persona que le presta ayuda era "no familiar" o era "servicio doméstico puertas adentro". Por último, en los hogares donde existían más de dos personas mayores dependientes, ayudadas por el mismo cuidador, se incluyó información únicamente del dependiente con mayor gravedad.

\section{Análisis estadístico}

Como se señaló, este estudio busca caracterizar a los cuidadores informales de personas mayores dependientes, y a su vez, evaluar el efecto de desempeñar este rol en la salud propia. Para ello, en primer lugar, se estimaron estadísticos descriptivos (porcentajes y medias), comparando las características de los cuidadores informales 
versus el resto de la población que no desempeña este rol.

En segundo lugar, se trabajó con la metodología de matching a través de propensity score, que es una técnica ampliamente utilizada para estimar efectos de tratamiento ${ }^{22}$. Básicamente, esta técnica produce dos grupos balanceados, uno de cuidadores y otro de no cuidadores. Para ello se calcula en primer lugar las probabilidades de ser cuidador informal, a partir de un modelo logístico, que en este caso incluyó las covariables: sexo, edad, estado civil, educación, ocupación e ingreso. Estos resultados son transformados en una puntuación (score) que resume la información de todas las covariables previamente mencionadas, y que es utilizada para seleccionar para cada cuidador (grupo "tratado") un individuo que posea una puntuación similar pero que no es cuidador (grupo "control o no tratado").

Finalmente, se estima el efecto promedio del tratamiento (average treatment effect), es decir, el efecto de ser cuidador informal versus no ser cuidador sobre la salud, medida a través de la pregunta s13. "En una escala de 1 a 7 , donde 1 corresponde a muy mal y 7 a muy bien, ¿qué nota le pondría a su estado de salud actual?". Dicha pregunta fue dicotomizada en 0: mala y regular salud (nota 1 a 5) y 1: buena o excelente salud (nota 6 y 7). La hipótesis detrás es que dado dos individuos cuyas características observables son tan similares entre sí como sea posible, cualquier diferencia en su percepción de salud es atribuible al efecto de proveer cuidado.

Para realizar estas estimaciones, se trabajó con el programa estadístico StataCorp. 2015 software estadístico Stata versión 14,(StataCorp LP, College Station, TX, USA), a través del comando teffects psmatch, el que permite estimar el efecto promedio del tratamiento (ATE) y el efecto promedio del tratamiento sobre el tratado (ATET) a partir de los datos emparejados por la metodología de propensity score. La diferencia entre usar ATE o ATET es que en el primer caso se busca emparejar todos los casos de una muestra (tanto los tratados como los controles), mientras que ATET solo busca emparejar los casos "tratados". Con ello, dado que en esta ocasión se está trabajando con una base de datos grande, es decir, con muchas observaciones, se optó por la segunda opción, es decir, se utilizó la opción ATET, con errores estándar robustos y con un caliper de 0,01 .

\section{Resultados}

Tal como previamente se señaló, 4.313 personas fueron clasificadas como cuidadores informales de personas mayores en CASEN 2015. De estos, $44,5 \%$ eran hijos/as y $33,6 \%$ parejas (Tabla $1)$. Se observó, además, que la mayoría de los cuidadores eran mujeres $(66,8 \%)$, con una media de edad de 56,5 años $(\mathrm{SD}=16,14)$. La mitad de estos cuidadores tenía pareja y un tercio era soltero/a. El 43,4\% tenía educación básica o menos, y solo $15,4 \%$ educación superior. En cuanto a la ocupación, la amplia mayoría $(61,1 \%)$ era inactivo, pero también destacaba que cerca de un tercio señalaba estar ocupado. En cuanto a la media de ingreso, esta era de \$305.877 ( $\mathrm{SD}=\$ 442.605)$. En todas estas variables sociodemográficas se observaron diferencias estadísticamente significativas entre los cuidadores informales y los no cuidadores (Tabla 2 ).

La Tabla 3 muestra las diferencias estandarizadas de las covariables utilizadas para la estimación del propensity score, comparando dichas diferencias antes y después del matching. Los resultados de la muestra emparejada indicaron que la coincidencia en el propensity score estimado equilibró las covariables. Las diferencias estandarizadas fueron todas cercanas a cero y las relaciones de varianza fueron cercanas a uno.

La Figura 1 también muestra el ajuste del matching, comparando la distribución del grupo cuidador y no cuidador antes y después del emparejamiento, observándose que los datos estaban equilibrados, por tanto, las distribuciones de los grupos control-tratamiento se equipararon.

Tabla 1. Parentesco cuidador informal con persona mayor dependiente $(n=4.313)$

\begin{tabular}{|lc|}
\hline & Porcentaje \\
\hline Pareja & $33,6 \%$ \\
\hline Hijo/a & $44,5 \%$ \\
\hline Yerno/Nuera & $5,8 \%$ \\
\hline Nieto/a & $5,0 \%$ \\
\hline Hermano/a & $3,6 \%$ \\
\hline Otro familiar & $7,6 \%$ \\
\hline
\end{tabular}


Efecto del cuidado informal en la salud de cuidadores de adultos mayores - M. B. Fernández et al

Tabla 2. Características cuidadores informales versus no cuidadores'

\begin{tabular}{|c|c|c|c|c|}
\hline & & $\begin{array}{l}\text { Cuidadores informales } \\
\qquad n=4.313\end{array}$ & $\begin{array}{l}\text { No cuidadores } \\
n=208.417\end{array}$ & p value \\
\hline Sexo & $\begin{array}{l}\text { Mujer } \\
\text { Hombre }\end{array}$ & $\begin{array}{l}2.880(66,8 \%) \\
1.433(33,2 \%)\end{array}$ & $\begin{array}{r}109.926(52,7 \%) \\
98.497(47,3 \%)\end{array}$ & $<0,05$ \\
\hline Edad & Media (SD) & $56,5(16,14)$ & $43,9(19,15)$ & $<0,05$ \\
\hline Estado civil & $\begin{array}{l}\text { Con pareja (casado/conviviente) } \\
\text { Separado/anulado/divorciado } \\
\text { Viudo/a } \\
\text { Soltero/a }\end{array}$ & $\begin{array}{r}2.366(54,9 \%) \\
397(9,2 \%) \\
168(3,9 \%) \\
1.382(32,0 \%)\end{array}$ & $\begin{array}{r}105.735(50,7 \%) \\
14.043 \quad(6,7 \%) \\
12.791 \quad(6,1 \%) \\
75.848(36,4 \%)\end{array}$ & $<0,05$ \\
\hline Educación & $\begin{array}{l}\text { Básica o menos } \\
\text { Media } \\
\text { Superior }\end{array}$ & $\begin{array}{r}1.866(43,4 \%) \\
1.776(41,3 \%) \\
662(15,4 \%)\end{array}$ & $\begin{array}{l}61.103(29,4 \%) \\
95.670(46,0 \%) \\
51.265(24,6 \%)\end{array}$ & $<0,05$ \\
\hline Ocupación & $\begin{array}{l}\text { Ocupados } \\
\text { Desocupados } \\
\text { Inactivos }\end{array}$ & $\begin{array}{r}1.534(35,6 \%) \\
143(3,3 \%) \\
2.636(61,1 \%)\end{array}$ & $\begin{array}{r}108.965(52,3 \%) \\
8.812(4,2 \%) \\
90.640(43,5 \%)\end{array}$ & $<0,05$ \\
\hline Ingreso ${ }^{2}$ & Media (SD) & $\$ 305.877(\$ 447.453)$ & $\$ 442.605(\$ 732.169)$ & $<0,05$ \\
\hline
\end{tabular}

${ }^{1}$ Se comparan datos de personas de 15 años o más. ${ }^{2}$ Corresponde al ingreso autónomo corregido.

Tabla 3. Diferencias estandarizadas muestra pre y post matching

\begin{tabular}{|lcccc|}
\hline & $\begin{array}{l}\text { Standardized Differences } \\
\text { Raw }\end{array}$ & Matched & Raw & $\begin{array}{c}\text { Matched } \\
\text { Mance Ratio }\end{array}$ \\
\hline Mujer & 0,2452 & $-0,0074$ & 0,9817 & 1,002 \\
\hline Edad & 0,6083 & 0,0244 & 0,8314 & 0,9623 \\
\hline Tiene pareja & $-0,0791$ & $-0,0397$ & 1,010 & 1,003 \\
\hline Educación básica & 0,2167 & $-0,0097$ & 1,156 & 0,9958 \\
\hline Inactivo & 0,4775 & $-0,0148$ & 1,451 & 0,9962 \\
Ingreso & $-0,2505$ & 0,0371 & 0,5424 & 1,225 \\
\hline
\end{tabular}

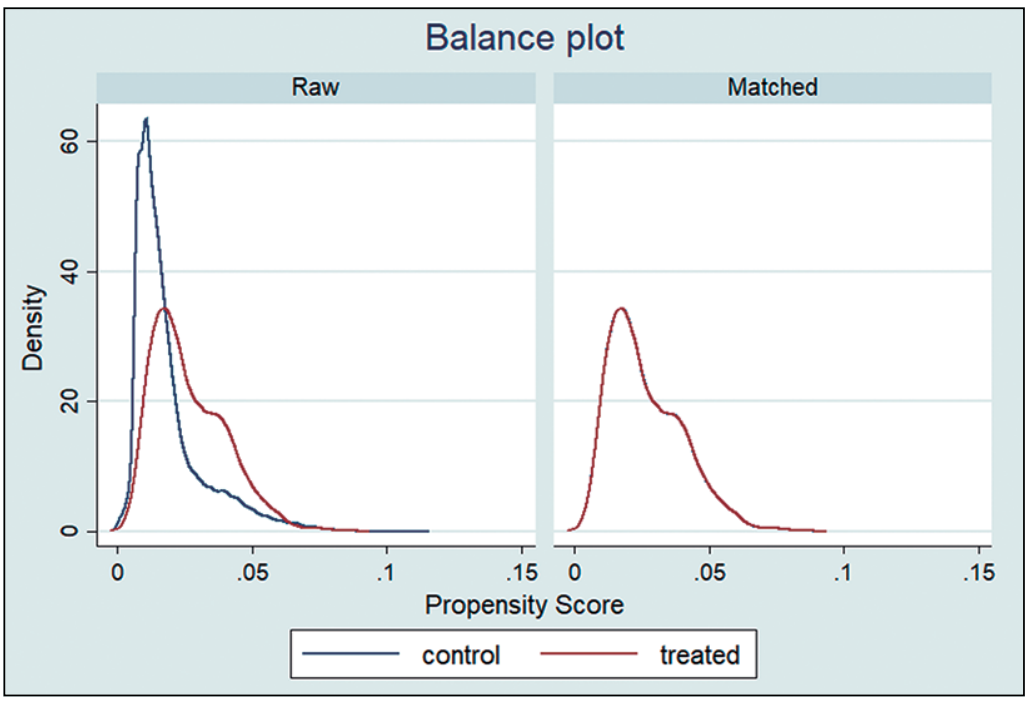

Figura 1. Gráfico de Densidad. 
Tabla 4. Efecto promedio del tratamiento (propensity score matching)

\begin{tabular}{|lcccc|}
\hline & Coef. & Error estándar & z & P-value \\
\hline ATET (es cuidador de persona mayor vs no es cuidador) & $-0,02509$ & 0,01221 & $-2,05$ & 0,040 \\
\hline
\end{tabular}

Finalmente, la Tabla 4 muestra el efecto promedio del tratamiento, es decir, el efecto de ser cuidador informal versus no serlo, sobre la autopercepción de salud. El ATET fue significativo estadísticamente $(\mathrm{p}<0,05)$ : ser cuidador informal de una persona mayor dependiente reduce la probabilidad de percibirse con una buena o excelente salud en comparación con una persona que no es cuidador.

\section{Discusión}

El objetivo de este estudio era, en primer lugar, describir quiénes son los cuidadores informales de personas mayor con dependencia en Chile. Tal y como otros estudios realizados en la región previamente lo evidencian, la responsabilidad de proveer cuidados recae principalmente en mujeres -hijas o parejas-, de mediana edad, con bajos niveles educativos y de ingresos y que no tienen un trabajo remunerado ${ }^{12,13,23,24,25}$. Con ello, se confirma la idea de que proveer cuidado informal no se distribuye de manera aleatoria en la población; más bien, las personas pertenecientes a ciertos grupos más vulnerables tienen mayor probabilidad de asumir dicha tarea.

Asimismo, una vez que se ha controlado este sesgo de selección, se confirma que proveer cuidado a una persona mayor dependiente tiene un efecto negativo sobre la salud de los cuidadores informales, lo que es consistente con otros estudios realizados en Europa y Estados Unidos de Norteamérica (EE.UU.) ${ }^{15,16,26,27}$. En el caso de Latinoamérica, hay que señalar que la mayoría de los estudios se han centrado, principalmente, en medir la asociación entre cuidado y salud del que cuida, siendo más bien escasos los estudios que evalúan el efecto causal entre ambos, con lo cual este artículo es un aporte a dicho vacío. Destaca el estudio de Hernández y Bigatti, quienes encuentran que el cuidar causa mayores niveles de depresión en una muestra de cuidadores mexicanos residentes en EE.UU. ${ }^{28}$.
En este punto, es importante destacar que, aunque en este estudio se utilizó solamente el indicador de percepción de la salud, es decir, solamente se trabajó con una variable subjetiva, distintos estudios han demostrado que existe una correlación entre este indicador y otros indicadores objetivos de salud, como la presencia de enfermedades crónicas o problemas de capacidad funcional ${ }^{29,30}$. De Zwart señala, además, que este indicador puede ser considerado como una medida que contiene en sí misma aspectos tanto de la salud física como mental ${ }^{15}$.

El cuidado es una tarea exigente, que produce elevados niveles de estrés, agotamiento emocional y sobrecarga en el cuidador, dando lugar a serias consecuencias para la salud. En el caso de la salud física, esta sobrecarga se asocia a la presencia de dolor muscular, seguido del cansancio físico y quejas cardiovasculares ${ }^{31,32}$. En el caso de la salud mental, se ve reflejado en una mayor presencia de problemas relacionados con ansiedad y depresión, pérdida de sensación de control y autonomía, peores estados anímicos, así como alteraciones en el sueño, apatía e irritabilidad ${ }^{25,33,34}$. En síntesis, el estado de salud global del cuidador se ve desfavorecido.

Avanzar, por tanto, en intervenciones que reduzcan el impacto negativo de cuidar es algo crucial, especialmente si se considera que el número de personas mayores seguirá en aumento. Los objetivos de estas deben apuntar, por una parte, a mejorar las competencias y estrategias de afrontamiento de los cuidadores, mediante, por ejemplo, capacitaciones en cuidados básicos, pero, también se debe reducir la cantidad de tiempo destinado al cuidado ${ }^{35}$. Si bien en Chile existen actualmente algunas iniciativas de esta naturaleza -tales como el Programa de Centros Diurnos del Adulto Mayor, el Programa de Cuidados Domiciliarios y el Fondo de Subsidio a los Establecimientos de Larga Estadía para Adultos Mayores-, la cobertura aún es limitada, por lo que los desafíos en materia de cuidado son algo en lo que aún se debe trabajar. 


\section{Referencias}

1. CASEN. Adultos Mayores: Síntesis de Resultados. Encuesta de Caracterización Socioeconómica Nacional 2015. Ministerio de Desarrollo Social. http://observatorio.ministeriodesarrollosocial.gob.cl/casen-multidimensional/casen/docs/CASEN_2015_Resultados_adultos_mayores.pdf.

2. CELADE Proyecciones de Población. Disponible en https://repositorio.cepal.org/bitstream/handle/11362/41018/1/S1600734_en.pdf.

3. Acosta E. Cuidados en crisis y mujeres migrantes hacia España y Chile. Dan más de lo que reciben. Bilbao: Ediciones Universidad de Deusto-Universidad Alberto Hurtado, 2015.

4. Colombo F, Llena-Nozal A, Mercier J, Tjadens F. Help Wanted? Providing and Paying for Long-Term Care. OECD Health Policy Studies. OECD Publising, 2011.

5. Jofré V, Sanhueza O. Evaluación de la sobrecarga de cuidadoras/es informales. Cienc Enferm 2010; 16 (3): 111-20.

6. Bom J, Bakx P, Schut F, van Doorslaer E. The Impact of Informal Caregiving for Older Adults on the Health of Various Types of Caregivers: A Systematic Review. Gerontologist 2018: 1-14.

7. Ruiz A, Nava M. Cuidadores: responsabilidades-obligaciones. Enf Neurol 2010; 3: 163-39.

8. Vélez J, Berbesí D, Cardona D, Segura A, Ordoñez J. Validación de escalas abreviadas de Zarit para la medición de síndrome del cuidador primario del adulto mayor en Medellín. Aten Primaria 2012; 44 (7): 411-6.

9. Pinquart M, Sörensen S. Differences between caregivers and noncaregivers in psychological health and physical health: A meta-analysis. Psychol Aging 2003; 18: 250-67.

10. Vitaliano $\mathrm{P}, \mathrm{Zhang} \mathrm{J}$, Scanlan J. Is caregiving hazardous to one's physical health? A meta-analysis. Psychol Bull 2003, 129: 946-72.

11. Aldana G, Guarino L. Sobrecarga, afrontamiento y salud en cuidadoras de pacientes con demencia tipo Alzheimer. Summa Psicol 2012; 9 (1): 5-14.

12. Cheix C, Herrera MS, Fernández MB, Barros, C. Factores de riesgo de la dependencia funcional en las personas mayores chilenas y consecuencias en el cuidado informal. América Latina Hoy 2015; 71: 17-36.

13. Rivera H, Dávila R, González A. Calidad de vida de los cuidadores primarios de pacientes geriátricos de la Clínica de Medicina Familiar Dr. Ignacio Chávez, ISSSTE. Rev Esp Med Quir 2011; 16 (1): 27-32.

14. Ávila J, Vergara M. Calidad de vida en cuidadores informales de personas con enfermedades crónicas. Aquichan 2014; 14 (3): 417-29.
15. de Zwart P. The effect of providing informal care on caregiver's health. Master Thesis. Erasmus University Rotterdam, 2015. https://thesis.eur.nl/pub/30834.

16. Stroka M. The Mental and Physical Burden of Caregiving - Evidence from Administrative Data. Ruhr Economic Papers 2014; 474.

17. Di Novi C, Jacobs R, Migheli M. The quality of life of female informal caregivers: From Scandinavia to the Mediterranean Sea. Eur J Popul 2015; 31: 309-33.

18. de Zwart P, Bakx P, van Doorslaer E. Will you still need me, will you still feed me when I'm 64? The health impact of caregiving to one's spouse. Health Econ 2017; 26 (2): 127-38.

19. Fukahori R, Sakai T, Sato K. The effects of incidence of care needs in households on employment, subjective health, and life satisfaction among middle-aged family members. Scott J Polit Econ 2015, 62: 518-45.

20. Schmitz H, Westphal M. Short- and medium-term effects of informal care provision on female caregivers' health. J Health Econ 2015, 42: 174-85.

21. Antonakis J, Bendahan S, Jacquart P, Lalive R. On making causal claims: A review and recommendations. Leadersh Q 2010; 21: 1086-120.

22. Rosenbaum P, Rubin D. The central role of the propensity score in observational studies for causal effects. Biometrika 1983; 70: 41-55.

23. Cardona D, Segura, A, Berbesí D, Ordoñez J, Agudelo A. Características demográficas y sociales del cuidador en adultos mayores. Investig Andina 2011; 13 (22): 17893.

24. Fernández MB, Lan Lay S. Multiple roles and subjective well-being of middle-aged women who are caregivers of elderly people in Chile. Journal of Women \& Aging 2018. https:/www.tandfonline.com/doi/full/10.1080/08 952841.2018.1537690

25. Slachevsky A, Budinich M, Miranda C, Núñez J, Muñoz C, Gloger S, et al. The CUIDEME Study: determinants of burden in chilean primary caregivers of patients with dementia. J Alzheimers Dis 2013; 35 (2): 297-306.

26. Heger D. The Mental Health of Children Providing Care to their Elderly Parent. Health Economics 2016; 12: $1617-29$.

27. Trivedi R, Beaver K, Bouldin E, Eugenio E, Zeliadt S, Nelson K, et al. Characteristics and well-being of informal caregivers: Results from a nationally-representative US survey. Chronic Illness 2013; 10 (3): 167-79.

28. Hernández A, Bigatti S. Depression among older Mexican American caregivers. Cultur Divers Ethnic Minor Psychol 2010; 16(1):50-8.

29. Ocampo J. Self-rated health: Importance of use in elderly adults. Colomb Med 2014; 41 (3): 275-89. 
30. Peláez E, Acosta L, Carrizo E. Factores asociados a la autopercepción de salud en adultos mayores. Revista Cubana de Salud Pública 2015; 41 (4): 638-48.

31. Gräsel E. When homecare ends-changes in the physical health of informal caregivers caring for dementia patients: a longitudinal study. J Am Geriatr Soc 2002; 50 (5): 843-51.

32. Jeanfaivre V, Diviné C, Akalin M, Anacharsis F, Montagne A, Le Parco J. A survey of pain prevalence at Albert-Chenevier Hospital. Comparison of patient, caregivers and physician assessments. Ann Med Interne
(Paris) 2003; 154: 499-508.

33. Savage $\mathrm{S}$, Bailey $\mathrm{S}$. The impact of caring on caregivers' mental health: a review of the literature. Aust Health Rev 2004; 27(1): 111-7.

34. Sherwood P, Given C, Given B, von Eye A. Caregiver Burden and Depressive Symptoms Analysis of Common Outcomes in Caregivers of Elderly Patients. J Aging Health 2005; 17 (2): 125-47.

35. Sörensen S, Pinquart M, Duberstein P. How effective are interventions with caregivers? An updated metaanalysis. Gerontologist 2002; 42: 356-72. 